Relations industrielles

Industrial Relations

\title{
Yves Bertrand et Patrick Guillemet, Les organisations: une approche systémique
}

\section{Laurent Bélanger}

Volume 46, numéro 4, 1991

URI : https://id.erudit.org/iderudit/050731ar

DOI : https://doi.org/10.7202/050731ar

Aller au sommaire du numéro

Éditeur(s)

Département des relations industrielles de l'Université Laval

ISSN

0034-379X (imprimé)

1703-8138 (numérique)

Découvrir la revue

Citer ce compte rendu

Bélanger, L. (1991). Compte rendu de [Yves Bertrand et Patrick Guillemet, Les organisations: une approche systémique]. Relations industrielles / Industrial Relations, 46(4), 875-876. https://doi.org/10.7202/050731ar

Tous droits réservés (C Département des relations industrielles de l'Université Laval, 1991
Ce document est protégé par la loi sur le droit d'auteur. L'utilisation des services d'Érudit (y compris la reproduction) est assujettie à sa politique d'utilisation que vous pouvez consulter en ligne.

https://apropos.erudit.org/fr/usagers/politique-dutilisation/ 
However, authors fail to point out the major problems the bargaining system faces in India; that unions in India are highly politicized and union leaders are mostly the outsiders, who are power hungry. There is a great deal of rivalry among unions and there are too many unions in a company in India. In Bombay, majority of plants are massively conflict prone. Unions often engage in slow-down tactics, i.e. in artificial restricting of output, whenever they want their demands to be met. Many employers are afraid of the power of unions.

While in Holland there is an extremely low level of pay dispersion, which indicates tight centralization of pay levels imposed by Phillip. In Bombay on the other hand, pay scales at two multinationals are determined by comparing the average total pay across the whole Bombay labour market regardless of their specific type of production or industrial classification.

As far as working hours and labour use is concerned there still remains areas of substantial inequality, i.e. considerably shorter working hours and longer sick leave in Holland than in Bombay. There are also major differences in overall management attitudes toward unions in India and Holland. The Phillips Company in Holland restricts bargaining with unions mostly to the issues of financial nature while in Bombay the management at Phillips Kalwa does negotiate with the unions, but does not always abide by the agreements.

In short, this study provides a detailed comparison of employment conditions and labour relations in the European and Indian plants of the same companies. The Headquarters of multinationals keep a tight control over decisions concerning restructuring and investment and isolate them from collective bargaining.

The book is easy to read and written in an interesting style. It is most thoroughly researched and serves as a useful guide to those interested in labour relations in international companies located in India and Holland.

Hem C. JAIN

University of New Brunswick

Les organisations: une approche systémique, par Yves Bertrand et Patrick Guillemet, les Éditions Agence d'Arc Inc., Chotard et associés, éds et Télé-Université, 1989, 333 p., ISBN 2-89022-164-4 (Les éditions Agence d'Arc Inc.)

Pendant longtemps, et suite aux enseignements de l'École de relations humaines, nous étions incités à définir les organisations sociales en retenant surtout et presque exclusivement les personnes et les rôles. Nous faisions alors des organisations «un regroupement d'individus accomplissant des tâches différenciées et coordonnées par la présence d'une hiérarchie en vue de réaliser un ou des objectifs communs». Dans ce présent ouvrage, qui sert de document de base pour un cours offert par Télé-université, les auteurs optent plutôt pour une conception beaucoup plus large des organisations en privilégiant l'approche systémique qui colle de près à l'analogie organiciste: cette tendance à comparer les organisations à des organismes vivants cherchant à survivre dans un environnement plutôt hostile. L'organisation devient donc pour ces deux auteurs «un système situé dans un environnement comprenant un sous-système culturel (des buts, fins, valeurs, croyances), un sous-système technoproductif (des connaissances, des technologies, de l'expérience), un sous-système structurel (une division formelle et informelle du travail), un sous-système psychosocial (des gens ayant des relations entre eux) 
ainsi qu'un sous-système de gestion (planification, contrôle, coordination)» (p. 7). En dépit de sa lourdeur, cette définition a l'avantage de faire ressortir la relation système et environnement, la nature de chacune des composantes importantes et leur état d'interdépendance.

L'articulation des chapitres obéit assez rigoureusement à cette définition. Après une brève présentation du contenu de leur ouvrage, les auteurs font d'abord un rappel de l'historique et des concepts de base de la théorie générale des systèmes pour s'en tenir dans un troisième chapitre à ceux qui caractérisent habituellement l'approche systémique: la notion de systèmes et les principes d'ouverture, de complexité, de finalité, de traitement, de globalité, de flux, de régulation, de rétroaction, d'équilibre et d'entropie. Ce chapitre se termine par une comparaison des approches analytique et systémique en s'appuyant sur l'ouvrage de Joël de Rosnay. Les autres chapitres abordent les composantes suivantes: la structure de l'organisation, l'environnement, la culture (buts, valeurs), les personnes, les groupes (sous-systèmes humains), le pilotage du système en termes de prise de décision, de planification stratégique et tactique. Un dernier chapitre, qui sert de conclusion, fait ressortir l'importance de la variable technologique comme dimension cognitive de l'organisation facilitant l'innovation et l'adaptation à l'environnement. En donnant ainsi à la systémique un caractère cognitif (approche socio-cognitive) et en l'appliquant à l'analyse de l'organisation, les auteurs font de cette dernière une «entité pensante». L'organisation est capable d'apprendre, c'est-à-dire, qu'elle peut s'adapter, régler des problèmes, inventer, imaginer, planifier, etc.» (p. 312).

Les auteurs ont réussi à rassembler avec succès une multitude de concepts et de propositions tirés de la systémique pour en faire une application intelligente et cohérente à l'étude des organisations. Cependant, en justifiant ainsi le paradigme systémique, ils se privaient à leur insu de l'apport conceptuel des autres approches, déjà bien décrites dans l'ouvrage de Gareth Morgan: Images de l'organisation. Ce dernier, tout en retenant l'analogie systémique, compare les organisations à une machine, à un cerveau, à une prison, à un flux ou à un processus de transformation. En faisant appel à plus d'un paradigme, on accroît ainsi la possibilité d'accéder à une meilleure compréhension de la réalité complexe des organisations actuelles.

Laurent BéLANger

Université Laval

Marchés du travail: limites sociales des nouvelles théories, par David Marsden, Paris, Édition Économica, 1989, 267 p., ISBN 2-7178-1756-5

Comme l'indique la jaquette à l'endos, l'ouvrage de David Marsden «évalue les tentatives d'un certain nombre d'économistes pour construire des liens entre la science économique, la sociologie du travail, et les relations professionnelles». L'objectif recherché est d'expliquer le fonctionnement du ou des, il faudrait plutôt dire pour rendre justice à l'auteur, marchés du travail. Dans sa préface, Jean-Jacques Sylvestre le qualifie de «l'ouvrage le plus clair, le plus rigoureux et le mieux informé en langue française sur cet important sujet". L'édition anglaise avait d'ailleurs été publiée en 1986 sous le titre The End of Economics Man? Custom and Competition in Labour Markets (St-Martin's Press, New-York).

Visant une approche pluridisciplinaire, l'auteur a réussi, en moins de 300 pages, à passer en revue, à évaluer et à critiquer la plupart des grandes théories économiques qui tentent d'expliquer les phénomènes des marchés du travail. Il y a ajouté des explications supplémentaires tirant leurs sources de la sociologie et des relations industrielles. Il a aussi fait des liens entre 